Maria Müller-Staub ${ }^{1}$ (Prof., PhD, EdN, RN), Nicole Zigan ${ }^{1}$ (MNS, RN), Daniela Händler-Schuster ${ }^{1}$ (Dr. rer. medic., RN), Sebastian Probst ${ }^{1}$ (DClinPrac, RN), Renate Monego ${ }^{2}$, Lorenz Imhof ${ }^{1}$ (Prof., PhD, RN)

${ }^{1}$ Zürcher Hochschule für Angewandte Wissenschaften ZHAW, Institut für Pflege, Departement Gesundheit, Winterthur, Schweiz

${ }^{2}$ Städtische Gesundheitsdienste Zürich, Schweiz

\title{
Umsorgt werden und umsorgen: Leben mit mehreren Langzeiterkrankungen (Leila) - Eine qualitative Studie zum Beitrag von APN in integrierter Versorgung
}

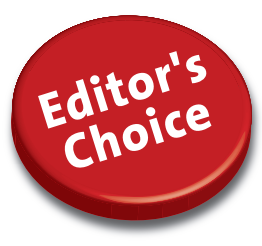

- Was ist (zu dieser Thematik) schon bekannt?

Pflegeexpert(inn)en APN tragen zur integrierten Versorgung bei. Die APN-Rollenimplementierung stellt jedoch Herausforderungen bezüglich der Übernahme und Gestaltung dieser neuen Rolle.

- Was ist neu? «Umsorgen», verbesserte Ressourcennutzung, therap. Regimes und Alltagsgestaltung charakterisieren APN-Leistungen bei chron. Kranken.

- Welche Konsequenzen haben die Ergebnisse für die Pflegepraxis? Die Zusammenarbeit mit Ärzt(inn)en ist auszuhandeln und Pflegeexpert(inn)en APN sind in die Spitex zu integrieren.

sen ergaben, dass die APN-Rollenanerkennung und -ausübung noch ausgehandelt werden muss. Zukünftige APN-Angebote für diese Patient(inn) engruppe sollten nebst Ärztenetzwerken in weitere Dienstleistungen und die Spitex integriert werden.

Schlüsselwörter: Advanced Practice Nursing (APN), Leben mit chronischen Krankheiten, multiple chronische Krankheiten, integrierte Versorgung, Grounded Theory

\section{Einleitung}

chronischen Krankheiten ist komplex und führt zu einer erhöhten Pflegeund Betreuungsbedürftigkeit. Zur Förderung der integrierten Versorgung wurde ein dreijähriges Projekt namens "Leben mit Langzeiterkrankung" (Leila) initiiert.

Ziel: Das Projekt hatte zum Ziel, Leistungen von Pflegeexpertinnen in Advanced Practice Nursing (APN) für chronisch erkrankte Menschen in Zusammenarbeit mit Hausarztpraxen zu entwickeln. Folgende Fragestellungen wurden untersucht: 1. Wie erleben Patient(inn)en, Zuweisende und Pflegeexpertinnen APN das Leila-Dienstleistungsangebot? 2. Wie gestalten sich Zuweisungsprozesse? 3. Wie erleben die Beteiligten die Zusammenarbeit und die APN-Rollenausübung?

Manuskript eingereicht am 30.12.2013 Manuskript akzeptiert am 16.5.2014
Methode: Es wurde ein qualitativer Ansatz gemäß Grounded Theory nach Corbin und Strauss gewählt, um Erfah rungen mit Leila und Interaktionen der an Leila beteiligten Personen zu untersuchen. Dabei wurden 38 Interviews mit Patient(inn)en, die mit multiplen chronischen Krankheiten leben, deren betreuende Pflegeexpertinnen APN und zuweisenden Ärzt(inn)en durchgeführt und evaluiert.

Ergebnisse: "Umsorgt werden und umsorgen" zeigte sich als zentrale Kategorie. Diese beschreibt wie auf die Patient(inn)en eingegangen wurde, sie einbezogen und ernst genommen wurden. Die Hauptkategorie "Den Alltag gestalten" zeigt, dass die Patient(inn)en lernten, mit den Folgen chronischer Erkrankungen im Alltag umzugehen. "Alle Ressourcen nutzen" legt dar, wie Potenziale und Stärken eingesetzt wurden.

Schlussfolgerungen: Die Ergebnisse zu Zuweisungs- und Kooperationsprozes-
Der komplexe Pflegebedarf von Patient(inn)en, die multimorbid und chronisch erkrankt sind, stellt für die Gesundheitsversorgung eine der größten Herausforderungen dar (World Health Organization [WHO], 2002; World Health Organization, 2005). Gerade die psychosozialen Anforderungen, welche ein Leben mit mehreren chronischen Krankheiten mit sich bringt, sind komplex und führen zu einer erhöhten Pflege- und Betreuungsbedürftigkeit (Saxe et al., 2007; Zuniga, Jenni, Wiesli \& Schwendimann, 2010). Chronische Erkrankungen zeigen oft psychosoziale Folgen, die von Seiten der Gesundheitsfachpersonen manchmal unterschätzt werden, was dazu führt, dass chronisch Erkrankte multiple Therapieangebote aufsuchen (Price, 1996; Saxe et al., 2007). In einer schweizerischen Studie gaben $66 \%$ von insgesamt 86 befragten Personen mit chronischen Erkrankungen an, in den letzten drei Monaten neben der ärztlichen Behandlung multiple Therapie- 
angebote aufgesucht zu haben. Dabei hatten drei Viertel der Erkrankten im Vorjahr eine medizinische Versorgung für eine schwerwiegende chronische Erkrankung erhalten. Von diesen Patient(inn)en hatten $91,7 \%$ in den vergangenen zwölf Monaten eine bis drei verschiedene Ärzt(inn)en besucht («International Health Policy Survey 2011» Sturny \& Camenzind, 2011). Die Betreuung multimorbider chronischer Patient(inn)en erfordert eine integrierte Versorgung, welche psychosoziale Aspekte einbezieht, komplexe Pflegebedarfe berücksichtigt und diverse Gesundheitsangebote koordiniert (Price, 1996; World Health Organization, 2005).

Zur Förderung einer integrierten Versorgung sind heute weltweit Advanced Practice Nurses (APNs) im Einsatz (DiCenso, Bourgeault et al., 2010). APNs sind Pflegende mit erweiterten Kompetenzen in klinischer Praxis, in Beratung, Patientencoaching und Forschungsanwendung (Hamric, Spross \& Hanson, 2008). Mittlerweile liegen viele positive Resultate zum Beitrag von APNs zu einer verbesserten Versorgung vor (Altersved, Zetterlund, Lindblad \& Fagerström, 2011; DiCenso \& Bryant-Lukosius, 2010; Lowe, Plummer, O'Brien \& Boyd, 2011). Internationalen Entwicklungen entsprechend wurden in der Schweiz seit den 1980er Jahren Pflegeexpert(inn)en ausgebildet, welche erweiterte pflegerische Aufgaben wahrnehmen. In Anlehnung an die Beschreibung des International Council of Nurses (ICN) wurde in der Schweiz folgende Definition formuliert: «Eine APN ist eine registrierte Pflegefachperson, welche sich Expertenwissen, Fähigkeiten zur Entscheidungsfindung bei komplexen Sachverhalten und klinische Kompetenzen für eine erweiterte pflegerische Praxis angeeignet hat». Die Charakteristik der Kompetenzen wird vom Kontext und/ oder den Bedingungen des jeweiligen
Landes geprägt, in dem sie für die Ausübung ihrer Tätigkeit zugelassen ist. Ein Masterabschluss in Pflege (MScN) gilt als Voraussetzung (Mahrer-Imhof, Eicher, Frauenfelder, Oulevey Bachmann \& Ulrich, 2012). Mittlerweile sind in der Schweiz seit zwölf Jahren Pflegeexpert(inn)en APN mit akademischen Masterabschlüssen im Einsatz. Bislang wurden diese jedoch vor allem in spitalinternen Bereichen eingesetzt (Ullmann-Bremi, Schluer, Finkbeiner \& Huber, 2011; Ulrich, Hellstern, Kressig, Eze \& Spirig, 2010; Zuniga et al., 2010).

Das Projekt «Leben mit Langzeiterkrankung» (Leila) wurde durch das Gesundheits- und Umweltdepartment der Stadt Zürich im Jahr 2009 zur Förderung der integrierten Versorgung initiiert. Das Projekt hatte zum Ziel, Leistungen von Pflegeexpertinnen APN für chronisch erkrankte Menschen in Zusammenarbeit mit Hausarztpraxen $\mathrm{zu}$ entwickeln. Zugleich sollte eine erste schweizerische Untersuchung zu Pflegeexpertinnen APN als Teil der integrierten Versorgung durchgeführt werden (Müller-Staub, Zigan, Probst \& Imhof, 2012). Leila fokussierte auf Selbstpflegeförderung und Edukation chronisch kranker Menschen unter Einbezug von APNs. Als Eckpfeiler des Projekts Leila galten: a) Die Leistungen werden in mindestens drei Gruppenpraxen erbracht; b) Trägerschaft der Leistungen sind die Städtischen Gesundheitsdienste Zürich; c) Leistungserbringer ist ein Team von drei Pflegeexpertinnen APN; d) Die Leistung «Begleitung in komplexen Situationen» wird vorwiegend in Hausbesuchen und in Zusammenarbeit mit anderen Diensten erbracht (wie Hausärzt(inn)en, Spezialist(inn)en, Akutspitäler, Langzeitinstitutionen, Spitex); e) Die Leistung «Beratung und Schulung zum bestmöglichen Umgang mit den Erkrankungen und ihren Auswirkungen im Alltag» findet in Einzel- und/oder Familiengesprächen sowie in Kleingruppen statt (Stadt Zürich Gesundheits- und Umweltdepartement, 2010). Die Haupttätigkeiten waren: Abklärung/klinisches Assessment; Motivierungs- und Schulungsarbeit; Begleitung/direkte Hilfestellungen; Mithilfe bei der Therapieoptimierung und Selbstmanagementempfehlungen. Der vorliegende Artikel soll Erfahrungen der Patient(inn)en bezüglich LeilaDienstleistungen, Kooperations- und Zuweisungsprozesse und die APN-Rollenausübung beschreiben, um Hinweise zum Einsatz von Pflegeexpertinnen APN in der integrierten Versorgung ableiten zu können. Es wurden folgende Forschungsfragen gestellt:

1. Wie erleben Patient(inn)en, Zuweisende und Pflegeexpertinnen APN das Leila-Dienstleistungsangebot?

2. Wie gestalten sich Zuweisungsprozesse?

3. Wie erleben die Beteiligten die $\mathrm{Zu}-$ sammenarbeit und die APN-Rollenausübung?

\section{Methode und Material}

\section{Forschungsmethode}

Im Zentrum der qualitativen Untersuchung standen das Erleben und die Erfahrungen der an Leila beteiligten Personen. Das Vorgehen der Studie orientierte sich daher am Ansatz der Grounded Theory (GT). Glaser und Strauss entwickelten die GT Methode als systematischen Ansatz für die Erforschung von Interaktionen (Corbin \& Strauss, 2008; Glaser, 1992). Die Grounded Theory Datenanalyse ist eine der am häufigsten verwendeten Methoden der Medizinsoziologie und der Pflege, um Prozesse im Bereich der Gesundheitsversorgung zu untersuchen. In einem ersten Schritt wurden in den transkribierten Interviewdaten erste Konzepte markiert, die erlaubten, die Bedeutung einzelner Prozesse für 
die Teilnehmenden sichtbar zu machen. In einem zweiten Schritt, einer induktiven thematischen Analyse, wurden dann die Eigenschaften dieser Kernkonzepte auf Gemeinsamkeiten und Differenzen genauer analysiert und dazu im Prozess eines fortlaufenden Vergleichs (constant comparison) Personen, Prozesse, Situationen und Interviewzeitpunkte miteinander verglichen. Diese Vergleiche ermöglichten, Strukturen und Prozesse auf einer abstrakteren Ebene darzustellen (Konzepte, Kategorien, Konstrukte). Da Datenanalyse und Datensammlung - wie in Grounded Theory üblich - parallel erfolgten, konnte die Datenanalyse durch die gezielte Datensammlung unterstützt werden (theoretical sampling). Der ganze Prozess und die einzelnen Analyseschritte sowie die Diskussionen innerhalb des Analyseteams und die Entwicklungsschritte des Modells wurden schriftlich dokumentiert (siehe Abschnitt Datenanalyse).

\section{Forschungsteilnehmende}

An der Studie nahmen insgesamt 29 Personen teil: 1) Patient(inn)en, welche Leila genutzt hatten $(n=20) ; 2)$ Pflegeexpertinnen APN des Projektteams $(\mathrm{n}=3)$ und 3) Ärzt(in)en, welche Patient(inn)en an Leila überwiesen hatten ( $\mathrm{n}=6$ Zuweisende). Die Auswahl der Patient(inn)en geschah mittels Zufallsstichprobe, diejenige der Zuweisenden mittels theoretischer Stichprobenbildung und $\mathrm{zu}$ gleich wurden alle drei an Leila mitarbeitenden Pflegeexpertinnen APN einbezogen (Charmaz, 2006; Corbin \& Strauss, 2008; Polit \& Beck, 2012). Die drei Personengruppen, mit denen insgesamt 38 Interviews geführt wurden, werden im Folgenden näher beschrieben.

\section{Patient(inn)en}

Von insgesamt 86 an Leila teilnehmenden Patient(inn)en wurden zufällig 20
Interviewpartner(inn)en ausgewählt. Anhand einer Randomisierungstabelle wurde aus der Liste der an Leila Teilnehmenden eine zufällige Stichprobe gebildet. Die Einschlusskriterien für Patient(inn)en an Leila waren: Personen mit multiplen chronischen Erkrankungen, die in Zürcher Stadtteilen mit einer geringen Ärztedichte sowie einem hohen Anteil an bildungsfernen Menschen und Migranten/-innen lebten. Zusätzlich musste eine Kombination einer chronischen Erkrankung mit mindestens einem erschwerenden Faktor wie fehlende Therapietreue (von den Zuweisenden definiert als Nicht-Erscheinen bei vereinbarten Arztbesuchen oder/und Nicht-Einhalten der Medikation/Selbstkontrollen wie Blutzuckermessungen), Klagen über Schwierigkeiten, Übergangssituationen, erschwerte Kommunikation oder Risikomedikation vorliegen. Von der Studie ausgeschlossen waren urteilsunfähige Personen mit Demenz und Personen, die kein Deutsch verstehen (Bonsack, Monego \& Schmidhauser, 2009).

Das durchschnittliche Alter der Interviewteilnehmenden lag bei 72 Jahren (SD 13,9, Median 76). Der Anteil der Frauen betrug genau $50 \%(n=10)$. Der Zivilstand war wie folgt: neun Personen lebten in Partnerschaft/verheiratet, fünf Personen waren geschieden/ getrennt, drei Personen waren verwitwet, zwei Personen waren ledig und bei einer fehlten die Angaben. Da es sich um Patient(inn)en mit multiplen chronischen Krankheiten handelte, wurden die medizinischen Diagnosen nach ICD-10 erhoben und nach Hauptdiagnosen geordnet. Die häufigsten Hauptdiagnosen waren: Hypertonie (Code I10.90) bei $44 \%$ der Teilnehmenden, gefolgt von Diabetes mellitus Typ 2 mit Adipositas (Code E11.90) bei $15 \%$ der Teilnehmenden) und Depression (Code F32.9) bei $13 \%$ der Teilnehmenden.

\section{Zuweisende}

Insgesamt nahmen 19 zuweisende Ärzt(inn)en an Leila teil. Die Zuweisenden beteiligten sich nicht alle gleich aktiv an Leila, was sich in der Zusammenarbeit mit der Projektgruppe sowie in einer unterschiedlich hohen $\mathrm{Zu}$ weisungsrate zeigte. Um die Erfahrungen von aktiven Leila Nutzern, die eng mit den APNs zusammenarbeiteten und solchen mit wenig Zuweisungen zu erfassen, wurden die Zuweisenden in zwei Gruppen unterteilt: Zuweisende, die mehr als zehn Patient(inn)en an Leila überwiesen hatten und solche mit weniger als zehn Zuweisungen. Aus beiden Gruppen wurden anschließend je drei Interviewpartner(innen) gewählt (Ziehung von geschlossenen Umschlägen).

\section{Pflegeexpertinnen APN}

Es wurden alle drei Pflegeexpertinnen APN, die an Leila beteiligt waren, befragt. Kriterien, um in Leila als Pflegeexpertin APN mitzuarbeiten waren: Diplomierte Pflegende mit mehrjähriger Berufserfahrung in der Pflege von Patient(inn)en mit komplexen chronischen Erkrankungen, Erfahrungen in der spitalexternen Pflege und Master in Nursing Science oder ähnliche Weiterbildung.

\section{Datensammlung}

Die Interviews erfolgten in einem Zeitraum von zwölf Monaten und fanden an den verschiedenen Arbeits- oder Wohnorten der Teilnehmenden statt. Patient(inn)en wurden zu Hause befragt, Pflegeexpertinnen APN und $\mathrm{Zu}$ weisende in den jeweiligen Gesundheitszentren/Arztpraxen. Um den Entwicklungsprozess in Leila zu berücksichtigen, wurden Zuweisende und Pflegeexpertinnen APN je zweimal im Rahmen narrativer Einzelinterviews während 60 Minuten mittels In- 
terviewleitfäden durch dieselbe Person befragt. Die Interviews wurden durch drei Personen der Abteilung Forschung des Instituts für Pflege, ZHAW Winterthur, durchgeführt und digital aufgenommen. Das Institut für Pflege war mit dieser Studie als externe Evaluation betraut. Eine Forschungsassistentin interviewte alle Patient(inn)en, eine wissenschaftliche Mitarbeitende mit Masterabschluss in Pflege befragte die APNs und eine wissenschaftliche Mitarbeitende mit Doktorat in Pflege interviewte die Zuweisenden. Mit den Zuweisenden wurden insgesamt zwölf Einzelinterviews und mit den APN sechs Einzelinterviews durchgeführt. Die Patient(inn)en wurden fortlaufend ab dem sechsten Monat nach Projektbeginn interviewt $(n=20)$. Es war geplant, 20 Interviews mit Patient(inn)en zu führen, um mindestens ein Viertel der an Leila Beteiligten einzuschließen. Da sich bereits nach der Hälfte der Interviews keine neuen Themen zeigten, musste die geplante Anzahl nicht erhöht werden. Dasselbe galt für die Interviews mit den Zuweisenden und den APNs. Somit wurden insgesamt 38 Interviews geführt. Leitende Fragen an Patient(inn)en waren: Gründe der Teilnahme an Leila, Erwartungen und Ziele, Beratungsverlauf, Durchführung und Wirksamkeit von Handlungen (pflegerische Maßnahmen, Selbstpflegehandlungen), Zufriedenheit mit dem Angebot und Kompetenzen der Pflegeexpertin APN. Dieselben Fragen wurden den Zuweisenden und Pflegeexpertinnen APN gestellt, und zugleich Zuweisungsgründe sowie Wirksamkeit und Nutzen der Leila-Interventionen erfragt. Die Interviewleitfäden mussten im Verlauf kaum angepasst werden. Die APNs beim zweiten Interview mehr über die Leila-Angebote als am Anfang des Projekts. Die Aussagen der Zuweisenden zu den zwei Interviewzeitpunkten zeigten hingegen kaum Unterschiede und wiesen viele Wieder- holungen auf. Die Tonbandaufnahmen der Interviews wurden durch die Interviewenden pseudonymisiert, der Forschungsassistentin zur Transkription übermittelt und von dieser ins Programm Atlas.ti 6.2.15 aufgenommen.

\section{Datenanalyse}

Die Interviews wurden zuerst pro Teilnehmendengruppe (Patient(inn) en, APNs, Zuweisende) durch je eine Forschende analysiert und codiert. Somit wurde jede Sichtweise (Patient(inn) en, APNs, Zuweisende) berücksichtigt. Während Zeile für Zeile analysiert wurde, konnten 453 Codes generiert und entsprechenden Textpassagen zugeordnet werden. Anschließend wurden die vorläufigen Ergebnisse vergleichend im Forschungsteam diskutiert. Weil die Analysen der Teilnehmendengruppen sehr ähnliche, bzw. sich deckende Kategorien und Subkategorien ergaben, wurden im dritten Schritt die aus den Analysen gewonnenen Kategorien des gesamten Datenmaterials (Patient(inn)en, Zuweisende, Pflegeexpertinnen APN) einer weiteren Analyse und einer abschließenden Zusammenfassung und Verifizierung unterzogen. Diese Verifizierung der Kategorien erfolgte parallel zur Datensammlung von einem wissenschaftlichen Mitarbeiter mit Doktorat und dem Leiter der Forschung und Entwicklung und beinhaltete im ersten Schritt «offenes Kodieren» (Charmaz, 2006). Im zweiten Schritt wurden aus den Codes durch «fokussiertes Kodieren» Kategorien und Subkategorien abgeleitet (Charmaz, 2006; vgl. axiales Kodieren Corbin \& Strauss, 2008) und die Methode der grafischen Darstellung (mapping) angewendet (Charmaz, 2006; Clarke, 2005). Bei der Kodierung der Hauptkategorien galten folgende Kriterien: 1. Hauptkategorien müssen zentral sein und eine Bedeutung für andere
Kategorien aufweisen; 2. Hauptkategorien müssen in den Daten fortwährend vorkommen; 3. Die Zusammenhänge $\mathrm{zu}$ anderen Kategorien sind bedeutungsvoll und ergeben sich einfach; 4. Hauptkategorien enthalten Implikationen für mögliche, abzuleitenden Annahmen; 5. Hauptkategorien bestätigen sich in den Daten konstant und 6. Hauptkategorien stellen Problemdimensionen des Forschungsgegenstands dar. Die Daten wurden solange analysiert und verglichen bis sich kohärente, durch die Daten laufend bestätigende Kategorien zeigten (Glaser, 1992). Dabei prüften vier Forschende den «Fit» aller Ebenen (Codes, Subkategorien, Hauptkategorien). Fit bedeutet Übereinstimmung der entwickelten Kategorien mit den Daten (Glaser, 1992) und erhöht Übertragbarkeit sowie Glaubwürdigkeit der Ergebnisse (Denzin \& Lincoln, 2005; Lincoln \& Guba, 1985).

\section{Ethische Betrachtungen}

Die Studie richtet sich nach dem Helsinki Protokoll (Institute of Clinical Research, 2007) und wurde durch die Kantonale Ethikkommission Zürich für unbedenklich erklärt (Entscheid KEK Zürich vom 11.05.2010). Alle Interviewteilnehmenden wurden über die Studienziele und das Vorgehen mündlich und schriftlich informiert und stimmten einer Teilnahme freiwillig zu.

\section{Ergebnisse}

Die Kernkategorie «Umsorgt werden und umsorgen» stellt das Hauptergebnis der Studie dar. Diese Kernkategorie sowie die Kategorien «Zuweisen» und «Zusammenarbeiten» stellten sich als Bedingungen für die Rollenausübung der Pflegeexpertin APN dar. 
Wie Patient(inn)en, Zuweisende sowie Pflegeexpertinnen APN das LeilaDienstleistungsangebot erlebten, kann anhand der Kernkategorie «Umsorgt werden und umsorgen» dargestellt werden. Diese Kernkategorie wird stark beeinflusst durch die zwei Hauptkategorien «Den Alltag gestalten» und «Alle Ressourcen nutzen». Als Bedingungen dafür, dass sich eine Pflegeexpertin APN in ihrer Rolle entwickeln kann, konnten die Bedingungskategorien «Zugewiesen werden und Zuweisen» und «Gut zusammen arbeiten» entwickelt werden (vgl. Abbildung 1). Alle Kategorien umfassen drei bis vier Subkategorien, die in den folgenden Ausführungen beschrieben werden.

\section{Kernkategorie "Umsorgt werden und umsorgen"}

Die Kernkategorie umfasst alle Teilnehmendengruppen und bildet $a b$, was Patient(inn)en Pflegeexpertinnen APN und Zuweisende unter den Begriffen «Umsorgt werden» und «umsorgen» verstehen. «Umsorgt werden» bedeutet, dass die Patient(inn)en in Leila Einfühlungsvermögen, Ehrlichkeit und Respekt sowie Wohlwollen erfuhren.
Zugleich bezeichneten Zuweisende und APNs Empathie, Kongruenz und Wertschätzung als zentralen und handlungsleitenden Schwerpunkt in Leila. Die Kernkategorie bedeutet, dass die APNs sowie die Zuweisenden auf die Patient(inn)en eingingen, sodass diese echtes Interesse für ihre persönlichen Anliegen, alltäglichen Sorgen und Emotionen beschrieben. «Umsorgen» steht für die professionelle Haltung der Pflegeexpertinnen APN und der $\mathrm{Zu}-$ weisenden, die sich im Leila-Angebot ausdrückte. «Umsorgt werden und umsorgen» bedeutet, sich als Patient(in) ernst genommen zu fühlen, und dass die Pflegeexpertinnen APN Patient(inn)enprobleme offen und hilfsbereit angehen. Die Zuweisenden bezeichneten das Eingehen auf die Patient(inn)en als die Hauptaufgabe der APN im Projekt Leila. «Es ist schön, dass sie gesagt hat: Sie können wieder anrufen, wenn irgendetwas wäre. Dann hat man immer einen Halt. Wenn irgendetwas wäre, kann man dort fragen und das finde ich schon gut.» (Patient(in), 1:132)

Aus dieser Kernkategorie konnten drei Subkategorien abgeleitet werden. Diese beschreiben die Sicht der Patient(inn)en

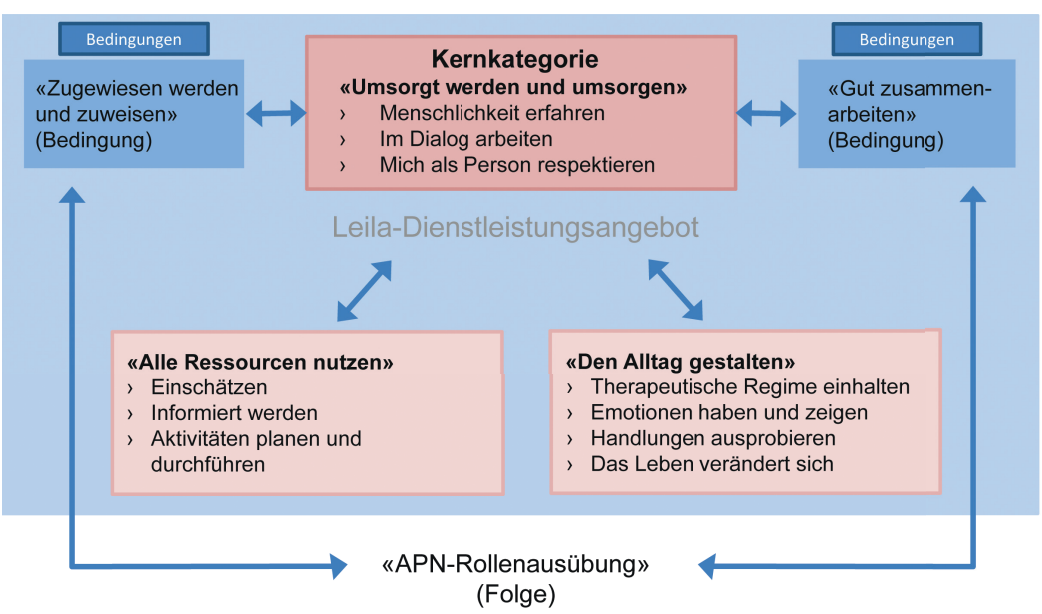

Abbildung 1: Ergebnisse: Haupt- und Kernkategorien sowie Zuweisen und Zusammenarbeiten als Bedingungen und APN-Rollenausübung als Folge. und wie sie ihre Pflege erlebten. Die Subkategorien sind: 1. «Menschlichkeit erfahren»; 2. «Im Dialog arbeiten» und 3. «Mich als Person respektieren».

\section{"Menschlichkeit erfahren"}

Menschlichkeit beinhaltet als zentrales Element fachkompetente Gesprächspartnerinnen zu haben, auf die man sich vertrauensvoll einlassen kann und von denen man Hilfe erfährt. «... Früher konnte man mit niemandem so intensiv über diese Dinge reden. Und eben Frau (Ärztin) hat gesagt, sie habe fast zu wenig Zeit für diese Dinge. Es ist schon schön, wenn man eine Ansprechperson hat, bei der man wei $\beta$, dass man alles fragen kann und es wird einem geholfen.» (Patient(in), 4:64)

\section{"Im Dialog arbeiten"}

Die Subkategorie «Im Dialog arbeiten» drückt aus, auf Augenhöhe partnerschaftlich als Patient(in) und Pflegeexpertin APN zusammenzuarbeiten und dabei Anregungen zum Umgang mit multiplen Erkrankungen zu gewinnen. Dies beinhaltet auch, dass die Patient(inn)en mit der Pflegeexpertin APN gemeinsame Ziele setzten.

«... Da versuche ich natürlich herauszufinden, wo das Potenzial von Veränderung wäre, wo sie darauf eingehen mögen und wo nicht.» (Pflegeexpertin APN, 5:48)

\section{"Mich als Person respektieren"}

«Mich als Person respektieren» bedeutet, als Patient(in) fachkompetente Gesprächspartnerinnen zu haben, welche die Patient(inn)en ernstnehmen, sodass sie wagen auch Schwieriges auszusprechen. Dazu gehört genügend Zeit für Beratungen und Gespräche an passenden, individuell gewählten Orten und in geschützten Räumen. Respektiert sein drückt aus, sich bei den Pflegeexpertinnen APN gut aufgehoben zu fühlen und Probleme offen aussprechen zu können. 
«... Man kann alles sagen, von dem man das Gefühl hat, dass man es rauslassen sollte». (Patient(in), 24:35)

\section{"Den Alltag gestalten"}

«Den Alltag gestalten» bedeutet, dass die Pflegeexpertinnen APN mit den Patient(inn)en Themen der Alltagsbewältigung bearbeiteten und viel Zeit mit den Patient(inn)en und in deren Alltag verbrachten. Die Alltagsgestaltung stellt eine der größten Herausforderungen für die Patient(inn)en dar. Entsprechend wurden Patient(inn)en dabei unterstützt, ihren Alltag trotz multiplen Krankheiten bestmöglich zu gestalten, Selbstpflege umzusetzen und mit Einschränkungen zu leben. Ebenso wurden gemeinsam Vorschläge dazu erarbeitet, wie alltägliche Ernährungs- oder Bewegungsgewohnheiten der Krankheitssituation entsprechend verbessert werden können. Die Komplexität der Alltagsgestaltung wird in vier Subkategorien beschrieben: 1 . «Therapeutische Regimes einhalten»; 2. «Emotionen haben und zeigen»; 3. «Handlungen ausprobieren» und 4 . «Das Leben veränderte sich».

\section{"Therapeutische Regimes einhalten"}

Der Begriff «Regime» bedeutet, Patient(inn)en so zu unterstützen, dass sie Behandlungspläne oder Methoden gesundheitsförderlicher Lebensgestaltung besser einhalten können. Diese Subkategorie beinhaltet, Therapieangebote besser zu verstehen und umzusetzen.

«Also wenn ein Patient ein wenig besser versteht, was er für eine Krankheit hat, nicht nur vom Arzt mitgeteilt, sondern auch vielleicht in andern Worten oder in einer anderen Form ... dass man so die Compliance verbessern kann». (Zuweisende(r), 3:16)

«... Sie (die Pflegeexpertin APN) hat mir aufgezeigt, dass ich mit dieser Krankheit leben muss. Dass ich die Krankheit im Griffhaben kann (...) dass ich mich nicht von der Krankheit beherrschen lassen muss oder diese verdränge; sondern, dass es ein gutes Leben mit der Krankheit geben kann.» (Patient(in) 2:28)

\section{"Emotionen haben und zeigen"}

«Emotionen haben und zeigen» bedeutet, vermehrt Gefühle zuzulassen und mitzuteilen. Diese Subkategorie beinhaltet die Auseinandersetzung mit multiplen Krankheiten und deren Folgen sowie einen offenen Umgang mit negativen Ereignissen oder Ängsten. Gemäß der Zuweisenden gewannen chronisch erkrankte Patient(inn)en durch die Pflegeexpertinnen APN mehr Selbstverstrauen, was sich darin zeigte, dass Patient(inn)en ihre Gefühle und Bedürfnisse vermehrt äußerten. «Leila versucht (...) wenn ein Patient kein Selbstvertrauen hat, das Selbstvertrauen des Patienten zu stärken (...) und seine/ihre Bedürfnisse auch sagen und aufzeigen zu können.» (Zuweisende(r), 4:93)

\section{"Handlungen ausprobieren"}

«Handlungen ausprobieren» beinhaltet, dass die Patient(inn)en durch die APNs zu Handlungen - wie z.B. neue Ess- oder Bewegungsgewohnheiten angeleitet wurden. Gemeinsam wurde festgelegt, Maßnahmen auszuprobieren und deren Wirkung zu reflektieren. «Also, ich bin (vorher) ja auch schon spazieren gegangen, aber durch Frau (Pflegeexpertin APN) realisierte ich den therapeutischen Nutzen. Ich begreife, dass es wichtig ist. Ich merke auch, dass es mir besser geht.» (Patient(in), 3:83).

\section{"Das Leben veränderte sich"}

Die Pflegeexpertinnen APN boten den Patient(inn)en laufend Gelegenheiten, über den Umgang mit multiplen Krankheiten zu sprechen. Sie wurden auch angespornt, über Zusammenhänge ihrer Verhaltensweisen und mögliche Konsequenzen nachzudenken. Die
Aussagen aus beiden Perspektiven bestätigen ein stärkeres Bewusstsein der Patient(inn)en für Handlungen bezüglich Gesundheitsförderung, sodass Patient(inn)en ihr Leben veränderten. Patient(inn)en kontrollieren Verläufe gezielter, stellen Einstellungsveränderungen fest und gestalten ihr Leben gesünder.

«Ja, ich habe Veränderungen vorgenommen; ich gehe mehr an die frische Luft und esse mehr Gemüse, also die Einstellung hat sich geändert.» (Patient(in), 3:107)

\section{"Alle Ressourcen nutzen"}

Die Kategorie «Alle Ressourcen nutzen» bedeutet, personale und Umfeld bezogene Potenziale und Stärken zu erkennen und einzusetzen. Dies beinhaltet, multiple Krankheiten «im Griff haben» und ressourcenorientiert Assessments durchzuführen, um Motivation und Stärken der Patient(inn)en zu eruieren. Zugleich geht es darum, Patient(inn)en vermehrte Informationen zu bieten und notwendige, weitere Therapieangebote zu wählen. $\mathrm{Zu}$ «Alle Ressourcen nutzen» gehören drei Subkategorien:

1. «Einschätzen»; 2. «Informiert werden» und 3. «Aktivitäten gemeinsam planen und durchführen».

\section{"Einschätzen»}

«Einschätzen» heißt, nach dem Befinden, den Wünschen und Zielen der Patient(inn)en zu fragen und Angaben zum Krankheitsverlauf und sozialen Umfeld zu erheben. Diese Situationseinschätzungen wurden von vielen Patient(inn)en als positiv gewertet, denn die Pflegeexpertinnen APN bauten ihre weiteren Beratungen darauf auf.

«Ein umfangreiches gerontologisches Assessment bei einer 91-jährigen Patientin zu machen heißt herauszufinden, wie sie mit ihrer Gebrechlichkeit vom Alter her in ihrem Alltag zurechtkommt 
und sie entsprechend $z u$ beraten.» (Pflegeexpertin APN, 8:22)

\section{"Informiert werden"}

«Informiert werden» bedeutet das Klären von Fragen zu Krankheiten, Medikamenten sowie zu Zusammenhängen zwischen Verhalten und Kranksein. Dazu gehören fachspezifische Informationen bezüglich Gesundheitsförderung oder Beratungsstellen und weiteren, unterstützenden Angeboten.

«Sie hat mir etwas erklärt wegen der Ernährung, auch wegen der Fußkontrolle und der Blutkontrolle.» (Patient(in), 10:21)

«Und Leila hat mir Vorschläge gemacht. Das ist eben unter anderem das 〈Diafit vom (Spital B). Das ist ganz neu (...) und dann hat sie mich dort angemeldet.» (Patient(in), 20:28)

\section{"Aktivitäten gemeinsam planen und durchführen"}

Diese Subkategorie umfasst die praktische und gemeinsame Planung und Durchführung von Handlungen des Selbstmanagements wie Bewegungsübungen oder Blutzuckerselbstmessungen und stellt dar, wie die APN zusammen mit den Patient(inn)en individuelle Maßnahmen umsetzten.

«... Und manchmal sind wir auch zusammen gegangen und sind nicht so schnell gelaufen, dann sind wir auch noch abgesessen, dort hatte es ein schönes «Bänkli〉 ...» (Patient(in), 23:46)

\section{Bedingungen und Folgen: APN-Rollenausübung}

Als Bedingungen für die Entwicklung von Leila und der Rollenausübung der Pflegeexpertinnen APN zeigten sich die zwei Kategorien: 1. «Zugewiesen werden und zuweisen» und 2. «Gut zusammenarbeiten» (Abbildung 1).

Da die Rolle der Pflegeexpertinnen APN eingangs des Projektes nicht klar definiert war, konnte auch nicht vorausgesagt werden, welche APN Kompetenzen eingebracht werden können. Ebenso war unklar, welchen Einfluss die Zuweisungsprozesse auf die Rollenausübung darstellen würden. Aus den Daten ging hervor, dass während des Projektverlaufs immer wieder Anpassungen im Leila-Angebot stattfanden, um den Patient(inn)en die Pflege zu bieten, die sie brauchten. Die Anpassungen waren durch projektbezogene und äußere Einflussfaktoren be dingt und die APN-Rolle wurde anfänglich seitens der Pflegeexpertinnen APN und der Zuweisenden als unklar bezeichnet. Aus den Ergebnissen zeigte sich, dass die Rollenausübung der Pflegeexpertinnen APN stark durch die Kategorien «Umsorgt werden und umsorgen», «Den Alltag gemeinsam gestalten», und «Alle Ressourcen nutzen» geprägt wurde. Die APN Rollenausübung zeigte sich als Folge der Kern- und Hauptkategorien und war bedingt durch Zuweisungen und $\mathrm{Zu}-$ sammenarbeit. Daher stellte sich die Kategorie «APN-Rollenausübung» als eigene Kategorie und als Folge der andern Kategorien dar. Die Kategorien «Zugewiesen werden und zuweisen» sowie «Gut zusammenarbeiten» werden im Folgenden vorgestellt.

\section{"Zugewiesen werden und zuweisen"}

«Zugewiesen werden» beschreibt Erfahrungen von Patient(inn)en sowie deren aktiven Teilnahmewunsch an Leila.

«Ja, es war ein Wunsch von mir, dass man etwas macht, dass man mich betreut. Da meine Eltern in (Land) sind und ich hier. Ich habe zwar per Telefon Rückhalt von der Familie, aber das ist nicht das Gleiche ...» (Patient(in), 2:74) «Zuweisen» beinhaltet Zuweisungsarten und -gründe. Die administrativen Askpekte der Zuweisung zeigten sich als unkompliziert, Anmeldungen liefen mittels dafür entwickelter Formulare, per Fax, E-Mail oder mündlich. «Zuweisen» steht dafür, dass bisherige Therapieangebote oder Hilfestellungen nicht ausreichten und ein pflegerisches Basis-Assessment erforderlich war.

«Also, in den überwiegenden Fällen sind das sehr komplexe Situationen, wo man während der Sprechstunde auch nach wiederholten Konsultationen merkt, dass irgendwie der Therapieverlauf nicht so läuft wie man eigentlich möchte und nicht so richtig versteht, an was es liegt. Zum Beispiel, man erklärte einem Patienten, wie man ein Medikament nehmen muss und es klappt irgendwie nicht. Oder, dass der Patient nicht in die Sprechstunde kommt, obwohl es nötig wäre. Oder wenn man merkt, dass die Zeit um einem Patienten etwas zu erklären in der Sprechstunde nicht vorhanden ist.» ( $\mathrm{Zu}-$ weisende(r), 3:39)

«Wenn ich das Gefühl hatte, dass hinter den Problemen von der Behandlung vor allem eine soziale Belastung stand, die durch Leila abgeklärt werden sollte.» (Zuweisende(r), 8:1)

\section{"Gut zusammenarbeiten"}

Diese Kategorie beschreibt Aspekte der Kommunikation und Koordination zwischen Zuweisenden und Pflegeexpertinnen APN. Sie stellte sich als eine Bedingung für die erfolgreiche Umsetzung von Leila dar. Aus Sicht der $\mathrm{Zu}$ weisenden bedeutet gute Zusammenarbeit, den Pflegeexpertinnen APN auf einfachem, schnellem Informationsweg Aufgaben abzugeben und Entlastung zu erfahren. Während Zuweisende Fragen zur Zusammenarbeit hauptsächlich mit Aussagen zum Informationsaustausch beantworteten sprachen die Pflegeexpertinnen APN vor allem über ihr Erleben der Zusammenarbeit. Zuweisende schätzten unkomplizierte Überweisungswege, z.B. mittels Fax, und sie erwarteten, gut über Ergebnisse klinischer Assess- 
ments informiert zu werden. Rückmeldungen nach APN-Interventionen - mittels Einträgen in die elektronische Krankengeschichte oder EMails - funktionierten aus Sicht der Zuweisenden sehr gut.

«Leila hat bei uns die Möglichkeit, in die Krankengeschichte hineinzuschauen und da werden sehr rege Einträge gemacht. Das heißt, wenn wir dann den Patienten wieder sehen, haben wir einen kurzen Eintrag, was gelaufen ist. Das ist sehr hilfreich, es finden auch Telefonate statt ...» (Zuweisende(r), 9:20)

Die Pflegeexpertinnen APN berichteten über unterschiedliche Erfahrungen in der Zusammenarbeit mit Zuweisenden. Wenn Zuweisende sich mit ihnen über Patient(inn)en austauschten und sie merkten, dass der Nutzen ihrer Arbeit gesehen wurde, bezeichneten sie dies als gute Zusammenarbeit. Gute Zusammenarbeit zeichnete sich auch durch Akzeptanz und Offenheit seitens Zuweisender gegenüber der APNRolle aus.

«Bei anderen Zuweisungen muss ich sagen, habe ich sehr viel Offenheit von ärztlicher Seite gespürt, große Dankbarkeit für die Unterstützung. Das waren vor allem Dinge, wie so ein bisschen Case Management wo sie (die Ärzte) sagen, ich habe nur eine Viertelstunde Zeit, ich bin sehr froh, dass sich jemand die Gesamtsituation anschaut und versucht, eine Stabilisierung von der Lebens- und Gesundheitssituation zu bekommen.» (Pflegeexpertin APN, 1:91)

Mangelhafte Zusammenarbeit zeigte sich in fehlenden Überweisungen oder geringem Austausch. Hinderlich war, wenn sich Pflegeexpertinnen APN in ihrer Arbeit nicht verstanden fühlten oder wenn sie merkten, dass ihre Beiträge zur Gesamtbehandlung der Patient(inn)en wenig Beachtung erhielten. Manchmal fühlten sie sich entgegen ihres eigenen APN-Verständnisses eingesetzt. Sie selbst verstehen ihre Rolle als Patient(inn)en zentriert, pflegespezifisch, kompetent und autonom.

«Ich würde das nicht als Zusammenarbeit bezeichnen. Oft habe ich den Eindruck, die weisen mir jemanden zu, den sie jetzt gerne loswerden möchten, weil der zu viel ihrer Praxiszeit verschlingt. Und dann möchten sie eigentlich in Ruhe gelassen werden - das ist mein Eindruck.» (Pflegeexpertin APN, 4:78)

\section{Diskussion}

In dieser Studie wurde untersucht, wie Patient(inn)en sowie Zuweisende und Pflegeexpertinnen APN das Leila-Angebot erleben. Zugleich sollte herausgefunden werden, wie sich Zuweisungsprozesse gestalten, wie die Beteiligten die Zusammenarbeit erfahren und wie die APN-Rollenausübung erfahren wird.

\section{Erleben des Leila-Dienstleistungs- angebots}

«Umsorgt werden» stellte für die Patient(inn)en das speziell Neue dar das sie erlebten. «Umsorgen und umsorgt sein» wird als «caring» bezeichnet und steht für pflegerische Haltung und professionelle Werte (Hanson, 2012; Reed, 2010). Gemäß Studien fokussiert die Medizin vermehrt auf Krankheitsbehandlungen und Techniken («curing»), während chronisch Kranke zunehmend fragen: Kümmert sich überhaupt jemand um uns? (Clark, 2004). Die Unterstützung im Erleben des Krankseins und das Verstehen von Krankheit sind für multimorbide Patient(inn)en von großer Bedeutung (Kitson, Marshall, Bassett \& Zeitz, 2012). Die Zuweisenden schätzten nicht nur die Leila-Hausbesuche und die zeitlichen Ressourcen der Pflegeexpertinnen APN, sondern auch, dass diese nah an und mit den Patient(inn) en arbeiteten. Patient(inn)en und $\mathrm{Zu}$ weisende bezeichneten die Hausbesu- che als wichtige, neue Dienstleistung. Die Pflegeexpertinnen APN gingen individuell auf die chronisch erkrankten Personen ein und führten Assessments als Grundlage gemeinsamer Zielformulierungen und entsprechende Pflegeplanungen durch (Gordon, 2011; Müller-Staub, Needham, Odenbreit, Lavin \& van Achterberg, 2008). «Umsorgt sein» beinhaltet auch die Vertretung der Patient(innen) durch die Pflegeexpertinnen APN. Das Einstehen für andere wird als «advocacy» bezeichnet und gehört zur APN-Rolle (Hamric et al., 2008). Die Förderung der Autonomie der Patient(inn)en und der vermehrte Einbezug in Behandlungsentscheide zeigten sich in der Kategorie «Menschlichkeit erfahren». Studien belegen bessere Ergebnisse bei Patient(inn)en, denen durch Pflegeexpertinnen APN mehr Zeit und psychologische Betreuung gewidmet wurde, als bei Patient(inn)en ohne APNDienstleistungen (Altersved et al., 2011; Brooten, Youngblut, Deatrick, Naylor \& York, 2003; Logue, 2002). Die Zuweisenden bezeichneten das umfassende Einschätzen des Pflegebedarfs als Teil eines notwendigen, pflegerischen APN Casemanagements. Vorgängige Studien belegten die Notwendigkeit pflegerischer APN Casemanagementfunktionen und deren Nutzen wurde nachgewiesen (Bachus, 1995; Berkman, Glass, Brissette \& Seeman, 2000; Bodenheimer, MacGregor \& Stothart, 2005; Brooten et al., 2003; Bryant-Lukosius, 2010; Bryant-Lukosius, Dicenso, Browne \& Pinelli, 2004; Newhouse et al., 2011).

Die Kategorien «Den Alltag bewältigen», «Alle Ressourcen nutzen» und «Das Leben veränderte sich» verweisen auf die Wirksamkeit der APN-Interventionen. Patient(inn)en zeigten ein erhöhtes Bewältigungsverhalten und äußerten, besser mit ihrem Kranksein umgehen zu können. Studien bestätigen, dass durch Pflegeexpertinnen 
APN betreute Patient(inn)en weniger Unsicherheiten zeigten als solche ohne APN-Dienstleistungen (Frank, 2003; McCorkle et al., 2009). Aus weiteren Studien geht hervor, dass APNs bei chronisch Kranken sowie bei depressiven Patient(inn)en - im Vergleich mit regulärer Pflege - wirksamere Pflege boten. Diese führten erweiterte Pflegeinterventionen durch und verbrachten dafür mehr Zeit mit den Patient(inn)en als Nicht-APNs (Hanrahan, Wu, Kelly, Aiken \& Blank, 2011; Neff, Madigan \& Narsavage, 2003). Patient(inn)en zeigten effektive «Lebensveränderungen» wie gezielte Einhaltung therapeutischer Regimes und erhöhte Selbstpflegefähigkeiten. Die Zuweisenden bestätigten den Nutzen der APN-Dienstleistungen und gaben an, positive Effekte bei Patient(inn)en wahrzunehmen. Diese Aussagen werden durch die Literatur gestützt (Alvarez, 2001; Brooten et al., 2003; Hoffman, 2001; Newhouse et al., 2011). Gezielte, individuell angepasste APN-Pflegeinterventionen, wie Patient(inn)enschulungen, Coaching und Ermutigungen, können in weniger Spitalaufenthalte und bessere Gesundheitszustände resultieren (Hoffman, 2001; Neff et al., 2003; Newhouse et al., 2011).

\section{Zuweisungsprozesse und Informationsaustausch}

Administrative Aspekte der Zuweisung und der Informationsaustausch stellten sich als unkompliziert dar. Gründe für eine Zuweisung an Leila waren gesundheitlich und sozial problematische Situationen oder fehlende Therapietreue. Patient(inn)en wurden auch überwiesen, wenn sie die Ressourcen der Zuweisenden überstiegen oder weil Zuweisende die APN-Angebote als eine Erweiterung der ärztlichen Dienstleistung sahen. Dass es dafür speziell ausgebildete Pflegeexpertinnen APN braucht, zeigte sich als unbestritten. Die Betreuungskontinui- tät multimorbider und chronisch erkrankter Menschen über zeitliche, institutionelle und professionelle Grenzen hinweg ist für die Qualität gesundheitlicher Dienstleistungen entscheidend. Daher empfahl die WHO die Förderung der integrierten Versorgung und den Einsatz von APN (World Health Organization [WHO], 2002, 2008). Integrierte Versorgung bedingt einen Patient(inn)en-zentrierten Ansatz sowie einen flüssigen, umfassenden Austausch relevanter Informationen mit anderen Gesundheitsdiensten (Kitson et al., 2012). Ebenso betont und verfolgt die Schweizerische eHealth Strategie den Austausch relevanter Informationen zwischen den verschiedenen Anbietern der Gesundheitsversorgung und bezeichnet einen flüssigen Informationsaustausch als Voraussetzung einer integrierten Versorgung (Eidgenössisches Departement des Innern, 2007; Müller-Staub, 2009).

\section{Erfahrungen der Zusammenarbeit und APN-Rollenausübung}

Eine gute Zusammenarbeit wirkte sich positiv auf die Patient(inn)en aus und trug zu «Umsorgt werden und umsorgen» bei. Die Literatur bestätigt, dass APNs wesentliche Beiträge zu Informationsvermittlung, Koordination und zur Gestaltung von Übergängen leisten, was von Patient(inn)en als fürsorglich bezeichnet wird (Bradway et al., 2012). Demgegenüber erfuhren die Pflegeexpertinnen APN seitens der Zuweisenden manchmal Zurücksetzung oder Unterordnung. Dort, wo Zuweisende die Kompetenzen der Pflegeexpertinnen APN nicht zu erkennen schienen, resultierte eine schlechtere Zusammenarbeit. Andererseits sahen Ärzt(inn)en einen Gewinn in der $\mathrm{Zu}$ sammenarbeit mit den Pflegeexpertinnen APN, wenn sie diese als entlastend empfanden oder wenn Patient(inn)en durch die APN-Interventionen therapeutische Regimes besser einhielten.
Es ist bekannt, dass sich die anfängliche Rollenimplementierung und Anerkennung von APNs schwierig gestalten kann (Faris, Douglas, Maples, Berg \& Thrailkill, 2010; O'Brien, Martin, Heyworth \& Meyer, 2008; O`Brien, Martin, Heyworth \& Meyer, 2009; Szekendi, 2007).

\section{Schlussfolgerungen und Empfehlungen für die Praxis}

Für Personen, die mit multiplen chronischen Krankheiten leben hat «Umsorgt werden» eine zentrale Bedeutung. «Umsorgen, erhöhte Ressourcennutzung, Einhaltung therapeutischer Regimes sowie verbesserte Alltagsgestaltung» charakterisieren APN-Leistungen bei dieser Patientenpopulation. Die Studie zeigte, dass ein gemeinsames Verständnis zu APN-Dienstleistungen und neuen Formen der gemeinsamen Patientenbetreuung sich erst etablieren muss. Während die Pflegeexpertinnen APN eine pflegerischeigenständige Rollenausübung anstrebten, zeigten Zuweisende teilweise andere Vorstellungen. Die Pflegeexpertinnen APN fühlten sich in erheblicher Abhängigkeit von den Zuweisenden und erlebten dies manchmal limitierend. Daher lassen sich folgende Empfehlungen ableiten:

- Die Ergebnisse weisen auf einen Informationsbedarf der Zuweisenden bezüglich APN-Kompetenzen hin. Um das Potential von Pflegeexpertinnen APN zum Einsatz zu bringen, sollten Informationsangebote für Ärzt(inn)en und weitere Dienste bezüglich APN-Ausbildung, Kompetenzen und Aufgaben gemacht werden.

- Die Zusammenarbeit zwischen Ärzteschaft und Pflegeexpertinnen APN sollte gemeinsam ausgehandelt werden, damit die Pflegeexpertinnen APN ihre Rolle weiter etablieren können.

- Angebote wie Leila könnten in die Spitex integriert werden. Dies hätte 
zum Vorteil, dass die APN-Rolle in die pflegerische Versorgung integriert und damit das Angebot für weitere Zuweisende (z. B. auch nicht-ärztliche Dienste) geöffnet werden könnte.

\section{Limitierungen, Schlussfolgerungen für die Forschung}

Der Ansatz der GT zeigte sich als geeignet, um Erfahrungen der an Leila Teilnehmenden $\mathrm{zu}$ erforschen und Kategorien und hauptsächliche Zusammenhänge zu konzipieren. Weil die Analysen der Teilnehmendengruppen sich deckende Kategorien und Subkategorien ergaben, wurden die aus den Analysen gewonnenen Kategorien des gesamten Datenmaterials (Patient(inn)en, Zuweisende, Pflegeexpertinnen APN) mehrfach analysiert und einer abschließenden Verifizierung unterzogen. Die Bezüge zu den unterschiedlichen Teilnehmendengruppen konnten im Rahmen der vorliegenden Publikation nicht vollumfänglich dargestellt werden. Die Nachvollziehbarkeit und Transferabilität wurden durch die Beschreibung der Analyseprozesse und die Wiedergabe von Zitationen bestmöglich gesichert (Charmaz, 2006; Corbin \& Strauss, 2008; Polit \& Beck, 2012). Die Konzeption der Studie berücksichtigte den Projektstatus von Leila. Dies bedeutete, dass die LeilaDienstleistungen während des Projekts entwickelt und laufend angepasst wurden. Anfänglich konnten die Pflegeexpertinnen APN ihre Interventionen nicht klar benennen und daher konnten diese nicht mittels Interventionsstudie geplant und auf ihre Wirksamkeit hin überprüft werden. Die Ergebnisse sind nicht auf andere APNSettings oder Patient(inn)engruppen übertragbar. Um den Nutzen und die Wirksamkeit von Pflegeinterventionen bei spezifischen Pflegediagnosen und pflegesensible Patientenergebnisse messbar nachzuweisen (Ackley \& Ladwig, 2014; Johnson et al., 2012), wird für zukünftige Projekte und deren Evaluation der Einsatz von Pflegeklassifikationen empfohlen (Moorhead, Johnson, Maas \& Swanson, 2013). Aus der Studie sich ergebende, weitere Forschungsfragen betreffen hinderliche und förderliche Faktoren und Strukturen für eine erfolgreiche APN-Rollenausübung im deutschsprachigen Raum, die Prävalenz von Pflegediagnosen bei multimorbiden, chronisch Kranken und Nachweise zur Wirksamkeit von APNPflegeinterventionen.

\section{Danksagung}

Der Dank der Autor(inn)en gilt allen Personen mit chronischen Erkrankungen, die an der Studie teilnahmen. Ohne die Mitarbeit der Patient(inn)en, der zuweisenden Ärzt(inn)en und des Projektteams wären weder die Studie noch das Projekt Leila möglich gewesen. Ebenso danken wir den Mitarbeitenden der ZHAW, die an dieser Studie mitwirkten: Jutta Dreizler (MScN, RN) und Stephanie Künzi (BScN, Stud. MSc, RN).

An Leila haben mitgearbeitet: Christine Reichart (MAS Gerontologie, RN), Stefania Bonsack (MScN, RN) sowie Margot Klein (MScN, RN) und Christine Rex (MScN, RN). Wir danken den Auftraggebenden Renate Monego und Claudia Eisenring, Direktorin Städtische Gesundheitsdienste Zürich für die Zusammenarbeit.

\section{Beiträge der einzelnen Autor(inn)en}

LI: Projektleitung, Planung und -konzeption (Principal Investigator) MMS: Co-Projektleitung, Datenerhebung und -analysen, Manuskript NZ: Datenanalyse und Manuskript SP: Datenanalyse und Manuskript, DHS Manuskript
Being cared for and caring: Living with multiple chronic diseases (Leila) - A qualitative study about APN contributions to integrated care Background: Living with multiple chronic diseases is complex and leads to enhanced care needs. To foster integrated care a project called "Living with chronic disease" (Leila) was initiated. Aim: The aim was to develop an Advanced Practice Nursing (APN) service in collaboration with medical centers for persons who are living with multiple chronic diseases. The following research questions were addressed: 1. What are patients' experiences, referring physicians and APNs with the Leila-Service? 2. How are referral processes performed? 3. How do the involved groups experience collaboration and APN role development?

Methods: A qualitative approach according grounded theory of Corbin and Strauss was used to explore the experiences with the Leila project and the interaction of the persons involved. 38 interviews were conducted with patients who are living with multiple chronic diseases, their APN's and the referring physicians.

Results: The findings revealed "Being cared for and caring" as main category. The data demonstrated how patients responded to their involvement into care and that they were taken as serious partners in the care process. The category "organizing everyday life" describes how patients learned to cope with the consequences of living with multiple chronic diseases. "Using all resources" as another category demonstrates how capabilities and strengths were adopted. 
Conclusions: The results of the cooperation- and allocation processes showed that the APN recognition and $A P N$ role performance have to be negotiated. Prospective APN-services for this patient population should be integrated along with physician networks and other service providers including community health nursing.

Key words: Advanced Practice Nursing (APN), living with chronic disease, multiple chronic disease, integrated care

\section{Literatur}

Ackley, B. J.; Ladwig, G. B. (2014). Nursing diagnosis handbook: An evidencebased guide to planning care (10 ed.). St. Louis: Mosby/Elsevier.

Altersved, E.; Zetterlund, L.; Lindblad, U.; Fagerström, L. (2011). Advanced practice nurses: A new resource for Swedish primary health-care teams. International Journal of Nursing Practice, 17 (2), $174-180$.

Alvarez, C. A. (2001). Noncompliance or human nature? Clinical Nurse Specialist: The Journal for Advanced Nursing Practice, 15 (2), 51- 51.

Bachus, K. (1995). Advanced registered nurse practitioners: preparation, roles and practice settings. Kansas Nurse, 70 (9), 3-4.

Berkman, L. F.; Glass, T.; Brissette, I.; Seeman, T. E. (2000). From social integration to health: Durkheim in the new millenium. Social Science and Medicine, 51 (6), 843-857.

Bodenheimer, T.; MacGregor, K.; Stothart, N. (2005). Nurses as leaders in chronic care. BMJ, 330 (7492), 612-613.

Bonsack, S.; Monego, R.; Schmidhauser, M. (2009). Leben mit Langzeiterkrankung (Leila): Begleitung, Beratung und Schulung als Teil der hausärztlichen Versorgung. Ein Projekt im Gesundheitsnetz 2025. (Version Oktober 2009). Zurich: Stadt Zürich, Städtische Gesundheitsdienste.
Bradway, C.; Trotta, R.; Bixby, M. B.; McPartland, E.; Wollman, M. C.; Kapustka, H.; ... Naylor, M. D. (2012). A qualitative analysis of an advanced practice nursedirected transitional care model intervention. [Research Support, N. I. H., Extramural Research Support, NonU.S. Gov't]. The Gerontologist, 52 (3), 394-407.

Brooten, D.; Youngblut, J. M.; Deatrick, J.; Naylor, M.; York, R. (2003). Patient problems, advanced practice nurse (APN) interventions, time and contacts among five patient groups. Journal of Nursing Scholarship, 35 (1), 73 - 79.

Bryant-Lukosius, D. (2010). The clinical nurse specialist role in Canada: forecasting the future through research. Can J Nurs Res, 42 (2), 19-25; discussion $26-18$.

Bryant-Lukosius, D.; Dicenso, A.; Browne, G.; Pinelli, J. (2004). Advanced practice nursing roles: development, implementation and evaluation. Journal of $\mathrm{Ad}-$ vanced Nursing, 48 (5), 519-529.

Charmaz, K. (2006). Constructing grounded Theory: A practical guide through qualitative analysis. London: Sage.

Clark, J. S. (2004). An aging population with chronic disease compels new delivery systems focused on new structures and practices. Nursing Administration Quarterly, 28 (2), 105- 115.

Clarke, A. (2005). Situational analysis Grounded theory after the postmodern turn. Thousand Oaks: Sage.

Corbin, J.; Strauss, A. (2008). Basics of qualitative research: Techniques and procedures for developing grounded theory. Los Angeles: Sage.

Denzin, N. K.; Lincoln, Y. S. (2005). Handbook of Qualitative Research. Thousand Oaks: Sage.

DiCenso, A.; Bryant-Lukosius, D. (2010). Clinical nurse specialists and nurse practitioners in Canada: A decision support synthesis. Ottawa: Canadian Health Services Research Foundation.

Eidgenössisches Departement des Innern, B. f. G. (Ed.). (2007). Strategie «eHealth» Schweiz. B.rn: Bundesamt für Gesundheit.

Faris, J. A.; Douglas, M. K.; Maples, D. C.; Berg, L. R.; Thrailkill, A. (2010). Job satisfaction of advanced practice nurses in the Veterans Health Administration. Journal of the American Academy of Nurse Practitioners, 22 (1), 35- 44.

Frank, D. I. (2003). Elderly clients` perceptions of communication with their health care provider and its relation to health deviation self care behaviors. Self-Care, Dependent-Care \& Nursing, 11 (2), $15-30$.

Glaser, B. (1992). Emergence versus forcing: Basics of grounded theory analysis. Mill Valley, CA: Sociology Press.

Gordon, M. (2011). Pflegeassessment Notes: Pflegeassessment und klinische Entscheidungsfindung. Bern: Hans Huber.

Hamric, A. B.; Spross, J. A.; Hanson, C. M. (2008). Advanced Practice Nursing An integrative approach (3rd ed.). St Louis: Elsevier.

Hanrahan, N. P.; Wu, E.; Kelly, D.; Aiken, L. H.; Blank, M. B. (2011). Randomized Clinical Trial of the Effectiveness of a HomeBased Advanced Practice Psychiatric Nurse Intervention: Outcomes for Individuals with Serious Mental Illness and HIV. Nursing Research and Practice, 2011, 840248. doi: 10.1155/2011/840248

Hanson, J. (2012). From me to we: Transforming values and building professional community through narratives. Nurse education in practice. doi: $10.1016 / \mathrm{j}$. nepr.2012.08.007

Hoffman, J. (2001). Broad disease management interventions: reducing health care costs for plan members with congestive heart failure. Disease Management \& Health Outcomes, 9 (10), $527-529$.

Institute of Clinical Research. (2007). Proceedings of the 28th annual conference of the institute of clinical research 2007. Bucks, UK: Institute of Clinical Research.

Johnson, M.; Moorhead, S.; Bulechek, G.; Butcher, H.; Maas, M.; Swanson, E. (2012). NOC and NIC linkages to NANDA-I and clinical conditions (3 ed.). Maryland Heights: Elsevier Mosby. Kitson, A.; Marshall, A.; Bassett, K.; Zeitz, K. (2012). What are the core elements of patient-centred care? A narrative review and synthesis of the literature from health policy, medicine and nursing. Journal of Advanced Nursing. doi: 10.1111/j.1365-2648.2012.06064.x 
Lincoln, Y. S.; Guba, E. G. (1985). Naturalistic inquiry. Newbury Park: Sage.

Logue, R. (2002). The impact of advanced practice nursing on improving medication adherence in the elderly: an educational intervention. American Journal for Nurse Practitioners, 6 (5), 9.

Lowe, G.; Plummer, V.; O'Brien, A. P.; Boyd, L. (2011). Time to clarify - the value of advanced practice nursing roles in health care. Journal of Advanced Nursing, 68 (3), $677-685$.

Mahrer-Imhof, R.; Eicher, M.; Frauenfelder, F.; Oulevey Bachmann, A.; Ulrich, A. (2012). Expertenbericht APN: Schweizerischer Verein für Pflegewissenschaft (VFP).

McCorkle, R.; Dowd, M.; Ercolano, E.; Schulman-Green, D.; Williams, A.-l.; Siefert, M. L.; ... Schwartz, P. (2009). Effects of a nursing intervention on quality of life outcomes in post-surgical women with gynecological cancers. [Randomized Controlled Trial Research Support, N.I.H., Extramural]. Psycho-Oncology, $18(1), 62-70$.

Moorhead, S.; Johnson, M.; Maas, M.; Swanson, E. (2013). Nursing Outcomes Classification (NOC): Measurement of health outcomes (4 ed.). St. Louis: Elsevier.

Müller-Staub, M. (2009). Preparing nurses to use standardized nursing language in the electronic health record. Studies in health technology and informatics: Connecting Health and Humans, 146, $337-341$.

Müller-Staub, M.; Needham, I.; Odenbreit, M.; Lavin, M. A.; van Achterberg, T. (2008). Implementing nursing diagnostics effectively: cluster randomized trial. Journal of Advanced Nursing, 63 (3), 291-301.

Müller-Staub, M.; Zigan, N.; Probst, S.; Imhof, L. (2012). Begleitforschung zum Projekt «Leben mit einer Langzeiterkrankung (Leila)» (pp. 99). Winterthur: Zürcher Hochschule für Angewandte Wissenschaften.
Neff, D. F.; Madigan, E.; Narsavage, G. (2003). APN-Directed Transitional Home Care Model: achieving positive outcomes for patients with COPD. Home Healthcare Nurse, 21 (8), 543-550.

Newhouse, R. P.; Stanik-Hutt, J.; White, K. M.; Johantgen, M.; Bass, E. B.; Zangaro, G.; .. Weiner, J. P. (2011). Advanced Practice Nurse Outcomes 1990-2008: A Systematic Review. Nursing Economic\$, 29 (5), $230-251$.

O`Brien, J. L.; Martin, D. R.; Heyworth, J.; Meyer, N. R. (2008). Negotiating transformational leadership: a key to effective collaboration. Nursing \& Health Sciences, 10 (2), $137-143$.

O〉Brien, J. L.; Martin, D. R.; Heyworth, J. A.; Meyer, N. R. (2009). A phenomenological perspective on advanced practice nurse-physician collaboration within an interdisciplinary healthcare team. Journal of the American Academy of Nurse Practitioners, 21 (8), 444-453.

Polit, D. F.; Beck, C. T. (2012). Nursing research: Generating and assessing evidence for nursing practice $(9$ ed.). Wolters Kluwer Health/Lippincott Williams \& Wilkins.

Price, B. (1996). Illness careers: the chronic illness experience. Journal of Advanced Nursing, 24 (2), 275 - 279.

Reed, S. M. (2010). A unitary-caring conceptual model for advanced practice nursing in palliative care. Holistic nursing practice, 24 (1), $23-34$.

Saxe, J. M.; Janson, S. L.; Dennehy, P. M.; Stringari-Murray, S.; Hirsch, J. E.; Waters, C. M. (2007). Meeting a primary care challenge in the United States: chronic illness care. Contemporary Nurse: A Journal for the Australian Nursing Profession, 26 (1), 94- 103.

Stadt Zürich Gesundheits- und Umweltdepartement. (2010). Leila - Leben mit Langzeiterkrankung http://www. gn2025.ch/projekte/leila/beschreibung [04.04.2012].
Sturny, I.; Camenzind, P. (2011). Erwachsene Personen mit Erkrankungen - Erfahrungen im Schweizer Gesundheitssystem im internationalen Vergleich. Auswertung des International Health Policy Survey 2011 des Commonwealth Fund im Auftrag des Bundesamtes für Gesundheit (BAG). Neuchâtel: Schweizerisches Gesundheitsobservatorium.

Szekendi, M. K. (2007). Communication among advanced practice nurses and physicians working in teams in an acute care setting. Ph.D., University of Illinois at Chicago, Health Sciences Center. http://search.ebscohost.com/login. aspx? direct $=$ true $\& \mathrm{db}=\operatorname{cin} 20 \& \mathrm{AN}=200$ 9982224\&site=ehost-live Available from EBSCOhost cin20 database. [accessed 03/08/2013]

Ullmann-Bremi, A.; Schluer, A. B.; Finkbeiner, G.; Huber, Y. (2011). The first steps of an ANP-team - challenges and chances of ANP-teams at the Children's University Hospital Zurich. Pflege, 24 (1), $21-28$.

Ulrich, A.; Hellstern, P.; Kressig, R. W.; Eze, G.; Spirig, R. (2010). Advanced Nursing Practice in daily nursing care: practice development of an acute geriatric Advanced Nursing Practice team. Pflege, 23 (6), $403-410$.

World Health Organization [WHO]. (2002). Innovative Care for Chronic Conditions: Building Blocks for Action. Geneva: WHO,

World Health Organization [WHO]. (2005). Chronic diseases and health promotion Geneva: WHO.

World Health Organization [WHO]. (2008). The global burden of diesase: 2004 update. Geneva: WHO.

Zuniga, F.; Jenni, G.; Wiesli, U.; Schwendimann, R. (2010). Development of the role of an advanced practice nurse in the long-term care of elderly people in Switzerland [German]. Pflege, 23 (6), $375-383$. 
Was war die größte Herausforderung bei Ihrer Studie?

Sichtweisen zur Rolle von Pflegeexpertinnen APN herauszukristallisieren.

Was wünschen Sie sich bezüglich der Thematik für die Zukunft?

Pflegeexpertinnen APN in verschiedenen Settings, um wichtige Beiträge für Patient(inn)en zu leisten.

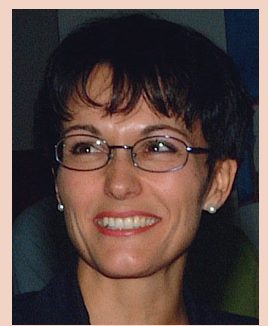

Maria Müller-Staub

Was empfehlen Sie den LeserInnen zum Weiterlesen/Vertiefen? «Improving patient care - the implementation of change in clinical practice» (Grol, Wening \& Eccles, 2010) und die «Nursing Interventions Classification» zum Benennen von Pflegeinterventionen (Bulechek, Butcher, Dochterman, \& Wagner, 2013)

\section{Korrespondenzadresse}

Nicole Zigan

Institut für Pflege

ZHAW Zürcher Hochschule für Angewandte Wissenschaften

Technikumstrasse 71

Postfach

8401 Winterthur

Schweiz

nicole.zigan@zhaw.ch 